presses like an accordion until the tapered tip of the needle passes through a flattened part of the silicone cap to rest within the bore of the syringe nozzle. This establishes in-line fluid continuity. When hydrostatic pressure in the infusion set exceeds that in the syringe nozzle during the process of withdrawal, some fluid in the set leaks back into the nozzle and drips off into the recess. This fluid remains there or evaporates until the next syringe nozzle is inserted. Owing to the relatively thin septum of flat silicone through which the tapered needle passes, some CLAVEs leak infusion fluid after repeated use and do so in proportion to the height of the water column above the port in a standing infusion set.

4. In the Baxter InterLink needleless IV system, the slit port caps are thick, do not leak with $>100 \mathrm{~cm}$ of retrograde hydrostatic pressure, and present no obvious recesses. However, when a blunt cannula (outside diameter $\pm 2.5 \mathrm{~mm}$ ) is withdrawn, the slit closes first on the infusion side, trapping fluid within the port cap. The amount of fluid trapped, albeit small and mostly squeezed out, probably is distributed evenly on both surfaces of the slit by the force of capillary action. This fluid is likely to remain there until the next blunt cannula is pushed through. All cannulae inserted subse- quently are obliged to pass through the same slit.

5. In all needleless IV systems, when the syringe or cartridge piston is not activated by manual control of the plunger, some fluid drips off the nozzle or blunt cannula into up-facing port recesses or slits. The volume dripping is proportional to the square of the radius of the leading bore.

6. Standard infusion sets with unslit infusion port caps are serviced by standard hollow-bore steel needles with sharp leading bevels and significantly smaller outside diameters, which seldom pass through the same track in the port cap; present no obvious recesses; seldom allow port cap leakage under more than $50 \mathrm{~cm}$ retrograde hydrostatic pressure; and are relatively efficient, because the same needle used to withdraw soluble medication from a vial is used to inject an infusion port. Needles currently are less costly than blunt cannulae, harpoons, and other paraphernalia essential to cannula use. Standard port caps require sharp needles, but the needle bores seldom are contaminated with blood or body fluids.

Thus, comparing needleless IV systems and standard infusion systems from a hydrodynamic point of view, it seems that standard systems are relatively safe, needed, efficient, and cost-effective for avoiding nosoco- mial BSI in patients. The added risk of standard systems for BSI from needlesticks in healthcare workers seems small in comparison. However, healthcare worker risks for BSI from sharp hollow-bore steel needles or needles used to insert catheters on the leading ends of needleless IV systems and standard infusion sets remain extreme.

\section{REFERENCES}

1. Voss A, Verweij P, L'Ecuyer PB, Fraser VJ. Needleless intravenous systems (with reply). Infect Control Hosp Epidemiol 1997;18:536,537.

2. McDonald LC, Banerjee S, Jarvis W. Central venous catheter (CVC)-associated bloodstream infections (BSI) in intensive care unit patients associated with needleless access devices. Infect Control Hosp Epidemiol 1997;18 (part 2):P24. Abstract 28

3. L'Ecuyer PB, Schwab EO, Iadomarco E, Barr N, Aton EA, Fraser VJ. Randomized prospective study of the impact of three needleless systems on needlestick injury rates. Infect Control Hosp Epidemiol 1996;17:803-808.

4. Danzig LE, Short IJ, Collins K, Mahoney M, Sepe S, Bland L, et al. Bloodstream infections associated with a needleless intravenous infusion system in patients receiving home infusion therapy. JAMA 1995;273:1862-1864.

Jack W. Shields, MD, MS Santa Barbara, California

\title{
Anergy Testing and TB Preventive Therapy for HIV-Infected Persons
}

\section{Gina Pugliese, RN, MS Martin S. Favero, PhD}

The $\mathrm{CDC}$ recently revised their recommendations for anergy skin testing and tuberculosis (TB) preventive therapy for HIV-infected persons. The report emphasizes that isoniazid (INH) preventive therapy is effective in reducing the incidence of active $\mathrm{TB}$ among persons who have HIV infection and latent TB. Because of the complications associated with TB disease in HIV-infected persons, these persons must be screened for TB infection. The $\mathrm{CDC}$ recommends that HIV-infected persons who have positive reactions to skin testing with purified protein derivative (PPD) tuberculin be evaluated to exclude active TB and offered preven- tive therapy with INH, if indicated. However, HIV-infected persons may have compromised ability to react to tuberculin skin testing, because HIV infection is associated with an elevated risk for cutaneous anergy.

Anergy testing is a diagnostic procedure used to obtain information regarding the competence of the cellular immune response. For anergy testing, the CDC recommends the use of two US Food and Drug Administration-approved Mantouxmethod tests (mumps and Candida), used together, with cut-off diameters of $5 \mathrm{~mm}$ of induration. Efforts to apply the results of anergy testing to preventive therapy decisions must be supplemented with information concerning the person's risk for infection with

\section{Mycobacterium tuberculosis.}

Factors limiting the usefulness of anergy skin testing include problems with standardization and reproducibility, the low risk for TB associated with a diagnosis of anergy, and the lack of apparent benefit of preventive therapy for groups of anergic HIV-infected persons. Therefore, the use of anergy testing in conjunction with PPD testing is no longer recommended for screening programs for $M$ tuberculosis infection conducted among HIV-infected persons in the United States.

FROM: Centers for Disease Control and Prevention. Anergy skin testing and preventive therapy for HIV-infected persons: revised recommendations. MMWR 1997;46(RR15):1-10. 